\title{
DISTRACTION POTENTIAL OF SPEECH-BASED DRIVER INTERFACES
}

\author{
John D. Lee, Kristi Schmidt, Toby Bral
}

University of Iowa - USA

\begin{abstract}
Summary: A common assumption concerning speech-based interaction with an invehicle information system is that the speech-based interaction does not distract driver, because the driver is not required to take his eyes off the road. This assumption does not take into consideration the cognitive demand placed on the driver. This cognitive demand may be highly dependent upon the nature of the interaction and may increase when errors occur in the interchange between the driver and the speech-based system. When the automatic speech recognition system makes an error, the driver must first recognize that an error has been made, determine how to recover from the error, trace back to the previous menu, and repeat the command to get the desired result. These additional steps and the error recovery process may place significant cognitive demands on driver. Understanding how these errors and the recovery process affects driver attention to the road is a critical design consideration for speech-based interaction with in-vehicle information systems. This paper describes an initial experiment to address this issue and provides a theoretical framework to help identify the requirements of a speech-interface needed to support easy error recovery. Because speech interactions will always be subject to human and system error, understanding how to support the robust interaction is critical in minimizing driver distraction.
\end{abstract}

